\title{
CONTROVERSY
}

\section{Antenatal treatment of a mother bearing a fetus with congenital adrenal hyperplasia}

\author{
C G D Brook
}

The virilisation of a female fetus by overproduction of adrenal androgens secreted by an adrenal gland with 21-hydroxylase deficiency is well known. Although reconstructive genital surgery can greatly improve matters, the long term outlook for reproduction is extremely poor for many reasons, including anatomy. ${ }^{12}$ The idea of prenatal treatment of 21hydroxylase deficiency by administering glucocorticoids to the mother to prevent virilisation was superficially extremely attractive. ${ }^{3}$ Where do we stand 15 years later?

\section{Treatment protocol and rationale}

The aim of treatment is to suppress the secretion of androgens from the adrenal gland of the affected female fetus because they will masculinise her external genitalia and probably, through conversion to oestrogen by aromatase, defeminise her brain and subsequent behaviour. In principle, the management protocol for a mother suspected of carrying a fetus affected with 21-hydroxylase deficiency, usually because she and her partner have had a previously affected child, is logical and straightforward.

Dexamethasone is started as soon as the pregnancy is confirmed and before virilisation can start. It is important to recognise that male sexual differentiation starts at about six weeks of gestation, and formation of the penis is complete at 14 weeks, so there is no time to lose.

It is necessary before conception to perform molecular studies on the proband and on both parents and also to confirm biochemically the heterozygote state of both parents to determine whether a molecular diagnosis will be possible. If it is, DNA can be isolated from a chorionic villous sample taken at between 10 and 12 weeks of gestation or from an amniotic fluid sample at $15-16$ weeks. It should be possible to determine quickly whether the fetus has the same molecular defect as the proband and what is its karyotype.

In the one in eight eventuality that the mother is carrying an affected female fetus, treatment with dexamethasone is continued until term. The aim is to deliver a female baby with normal genitalia, or, at very least, with a degree of virilisation much less than that seen in other affected family members.

\section{Genetic pitfalls}

Nothing in genetics is as straightforward as it seems at first sight and this is certainly true of 21-hydroxylase deficiency. The underlying molecular pathology of the system is extremely complex with gene and pseudogene, sometimes in multiple copies, located within the major histocompatibility complex on chromosome 6, along with genes for various components of the complement system.

Mutation analysis is not easy, especially in congenital adrenal hyperplasia, and unaffected parents can appear genotypically identical with their offspring ${ }^{4}$ (through the finding of multiple mutations on single alleles ${ }^{56}$ and non-amplification of alleles $\left.{ }^{7}\right)$. Even more puzzling is the observation that patients can have the disease without demonstrable genetic mutations. $^{8}$

In the face of these mismatches of genotype and phenotype, there is an absolute need for postnatal verification of a diagnosis made antenatally. We need to think carefully about both the mother and the fetus and about the very serious ethical issue of treating seven of eight pregnancies unnecessarily, if only to 12-14 weeks of gestation, because the fetus is unaffected, heterozygote, or male (number needed to treat $^{9}=8$ ).

\section{Effects on the mother}

Doses of dexamethasone have usually been large: $20 \mu \mathrm{g} / \mathrm{kg} / 24 \mathrm{~h}$ (equivalent to $0.7 \mathrm{mg}$ / $\mathrm{m}^{2} / 24 \mathrm{~h}$ ) in three divided doses has been recommended in the United States for reasons that are not entirely clear as the dose needed overnight to suppress adrenal function is 0.3 $\mathrm{mg} / \mathrm{m}^{2}$ body surface area. ${ }^{10}$ Consequently rather frightening side effects (weight gain, oedema, hypertension, glucose intolerance, hirsutism, and striae among others) have been reported, ${ }^{11}$ which have been seen to a much lesser extent in the clinical practice at our centre where we have used $0.25 \mathrm{mg}$ three times daily (about $0.5 \mathrm{mg} / \mathrm{m}^{2} / 24 \mathrm{~h}$ ). As maternal Cushing syndrome has a bad track record for the mother and the baby, ${ }^{12}$ we need to recognise the dangers of maternal complications at these sorts of doses.

Early amniocentesis (at 10-14 weeks of gestation) is slightly riskier in terms of miscarriage (3\% less chance of successful pregnancy 
outcome) than amniocentesis performed at the conventional time (15-16 weeks), when the risk of miscarriage is about $1 \%$. The overall risk of complications with either procedure is about $5 \% .{ }^{13}$ Amniocentesis performed at the conventional time is not only slightly safer but is also more accurate for fetal karyotyping than chorionic villous sampling because amniotic fluid samples are altered about 10 times less often by inherent chromosomal mosaic problems than villous samples.

Chorionic villous sampling, which increases the risk of pregnancy loss compared with amniocentesis performed at the conventional time by $0.5-4 \%,{ }^{13}$ comes into its own for the diagnosis of genetic disorders because it yields large amounts of DNA. Ideally the procedure should be performed at 10-12 weeks of gestation, and certainly not before 10 weeks because of the up to $2 \%$ risk of associated fetal limb defects. ${ }^{13}$

\section{Effects on the fetus}

The outcome of this treatment has generally been favourable in terms of improving genital ambiguity, but still about half of the affected treated females have needed surgical attention to their genitalia. ${ }^{14}$ This may partly be because dexamethasone has variable effects on fetal adrenal function. ${ }^{15}$ There must also be tissue response variability too because not all babies with classical congenital adrenal hyperplasia have ambiguous genitalia at birth. ${ }^{16}$ The timing of treatment is particularly relevant: a late onset or an inadequate dose of dexamethasone may prevent clitoral enlargement but may not prevent the formation of a urogenital sinus, which is a serious congenital abnormality with very adverse long term consequences. ${ }^{17}$

Babies in utero are generally protected against exposure to their own and their mother's cortisol, and fetal concentrations of cortisol are much lower than those of their mothers through a number of mechanisms. It is precisely to override this protection that dexamethasone, which is a synthetic fluorinated steroid, is used-for example, for the treatment of fetuses at risk of hyaline membrane disease with steroids to induce lung maturation. It is also used to suppress adrenal androgen secretion in babies with congenital adrenal hyperplasia. We can expect that there will be side effects of such treatment. How serious are they, particularly for the seven of eight fetuses who are treated unnecessarily during the first trimester?

Treatment of rats with low doses of dexamethasone throughout pregnancy reduces birth weight and produces persistently elevated blood pressure when the rats reach adult life. ${ }^{18}$ This is probably mediated through changes in the renal vasculature. The studies of Barker et $a l^{19}$ show how dangerous this could be for humans.

There has been only one good study of the long term effects of antenatal treatment of congenital adrenal hyperplasia: it comes from Sweden and makes scary reading. ${ }^{20}$ Fetal and childhood growth were generally satisfactory but adverse events were reported in both children treated just in the first trimester and those treated for the whole of gestation. Of 19 unaffected children, one boy treated for seven weeks had agenesis of the corpus callosum and another had delayed psychomotor development. Failure to thrive was reported in three unaffected girls, and one further girl had unexplained mood swings, so there was something to remark in about one third of the group. In the group of six affected girls treated for the whole of gestation, three were considered to have adverse events, even though only one had serious long term consequences.

\section{Conclusion}

There is no doubt that antenatal treatment of a mother bearing a fetus with congenital adrenal hyperplasia is effective in reducing genital ambiguity - but at a price. The price includes chorionic villous sampling and/or amniocentesis with their attendant risks, the problems associated with molecular diagnosis, and the unknown long term effects of exposing seven of eight babies to unnecessary treatment.

I find myself sobered by what I have reread during the preparation of this article, even though I know full well the extent of the continuing disaster of genital ambiguity in affected females. Like previous authors, ${ }^{21}{ }^{22} \mathrm{I}$ do not think that we should abandon the idea, but antenatal treatment should, like marriage, not be "enterprised, nor taken in hand, unadvisedly, lightly or wantonly". It needs the fullest of informed consent; it should only be undertaken by experts; it should be coupled with very long term follow up; it should only be undertaken in the context of large (probably international) collaborative trials.

I thank Dr G Rumsby for her guidance through the molecular maze, Professor Charles Rodeck for his advice, and Dr Stephen Herman for common sense.

1 Mulaikal RM, Migeon CJ, Rock JA. Fertility rates in female patients with congenital adrenal hyperplasia due to 21-hydroxylase deficiency. $N$ Engl f Med 1987;316:17821-hyd.

2 Premawardhana LDKE, Hughes IA, Read GF, Scanlon MF. Longer term outcome in females with congenital adrenal hyperplasia: the Cardiff experience. Clin Endocr 1997;46:327-32.

3 David M, Forest MG. Prenatal treatment of congenital adrenal hyperplasia resulting from 21-hydroxylase deficiency. F Pediatr 1984;105:799-801

4 Rumsby G, Massoud AF, Avey CJ, Brook CGD. Nonexpression of a common mutation in the 21-hydroxylase gene: implications for prenatal diagnosis and carrier testing. F Med Genet 1996;33:798-9.

5 Wedell A, Thilen A, Ritzen EM, Stengler B, Luthman H. Mutational spectrum of the steroid 21-hydroxylase gene in Sweden; implications for genetic diagnosis and association with disease manifestations. 7 Clin Endocrinol Metab 1994;78:1145-52.

6 Rumsby G, Avey CJ, Conway GS, Honour JW. Genotypephenotype analysis in late-onset 21-hydroxylase deficiency in comparison to the classical form. Clin Endocrinol 1998;48:707-11.

7 Day DJ, Speiser PW, Schultze E, et al. Identification of nonamplifying CYP21 genes when using PCR-based diagnosis of 21-hydroxylase deficiency in CAH affected pedigrees. Hum Mol Genet 1996;5:2039-48.

8 Nimkarn S, Cerame BI, Wei J-Q, et al. Congenital adrenal hyperplasia without demonstrable genetic mutations. I Clin Endocrinol Metab 1999;84:378-81.

9 Cook RJ, Sackett DL. The number needed to treat: a clinically useful measure of treatment effect. BMF 1995;310:452-4.

10 Hindmarsh PC, Brook CGD. Single dose dexamethasone suppression test in children: dose relationship to body size. Clin Endocrinol (Oxf) 1985;23:67-70.

11 Levine LS, Pang S. Prenatal diagnosis and treatment of congenital adrenal hyperplasia. $\mathcal{f}$ Pediatr Endocrinol 1994;7:193-200. 
12 Büscher MA, McClamrock HD, Adashi EY. Cushing's syndrome in pregnancy. Obstet Gynecol 1992;79:130-7.

3 Jauniaux E, Rodeck C. Use, risks and complications of amniocentesis and chorionic villous sampling for prenata diagnosis in early pregnancy. Biol Med 1995;1:245-52.

14 Mercado AB, Wilson RC, Cheng KC, Ji-Qing Wei, New MI. Prenatal treatment and diagnosis of $\mathrm{CAH}$ owing to steroid 21-hydroxylase deficiency. F Clin Endocrinol Metab 1995;80:2014-20.

15 Malunowicz EM, Ginalska-Malinowska M, Romer TE, Bal $\mathrm{K}$. The influence of prenatal dexamethasone treatment on urinary excretion of adrenocortical steroids in newborns. Eur F Pediatr 1998;157:539-43.

16 Quercia N, Chitayat D, Babul-Hirii R, Niw MI, Daneman D. Normal external genitalia in a female with classical CAH who was not treated during embryogenesis. Prenat Diagn 1998;18:83-5.
17 Couper JJ, Hutson JM, Warne GL. Hydrometrocolpos Couper JJ, Hutson JM, Warne GL. Hydrometrocolpos 7 Pediatr 1993;152:9-11.

18 Benediktsson R, Lindsay R, Noble J, Seckl JR, Edwards CRW. Glucocorticoid exposure in utero: a new model for adult hypertension. Lancet 1993;341:339-41.

19 Barker DJP, Gluckman PD, Godfrey KM, Harding JE, Owens JA, Robinson JS. Fetal nutrition and cardiovascular disease in adult life. Lancet 1993;341:938-41.

20 Lajic S, Wedell A, Bui T-H, Ritzen EM, Holst M. Long-term somatic follow-up of prenatally treated children with CAH. F Clin Endocrinol Metab 1998;83:3872-80.

21 Seckl JR, Miller WL. How safe is long-term prenatal glucocortucoid treatment? $\mathcal{F} A M A$ 1997;277:1077-9.

22 Kelnar CJH. CAH: the place for prenatal treatment and neonatal screening. Early Hum Dev 1993;35:81-90.

\section{Commentary}

Is there an element of unjustified scaremongering in this review which appears to place undue emphasis on the dangers of antenatal treatment for congenital adrenal hyperplasia? Intuitively, the rationale for treatment could not be more straightforward-use the negative feedback loop of classic endocrine function by replacing fetal cortisol production with exogenous glucocorticoid to inhibit adrenocorticotrophin induced excess fetal adrenal androgen production. However, the tricompartmental maternoplacental-fetal unit makes implementation of the loop in reality less straightforward. Nevertheless, David and Forest ${ }^{1}$ achieved a remarkable result when they showed for the first time that antenatal dexamethasone treatment given to the mother early in pregnancy could result in normal external genitalia in a female infant with congenital adrenal hyperplasia. That fortunate person is now a normally developed and healthy young adult. A major congenital malformation can be prevented with antenatal medical treatment.

Congenital adrenal hyperplasia is a lifelong endocrine disorder, the understanding and management of which involves a complex interplay of factors: genetic mechanisms; steroid production, action, and metabolism; pharmacokinetics of steroid formulations; growth, puberty, and reproduction; surgical reconstructive techniques; psychosexual development.

How beneficial would antenatal treatment be for the immediate and later management of congenital adrenal hyperplasia? There is every expectation that masculinisation of the female external genitalia can be prevented by treatment with judicious doses of dexamethasone given to the mother from early pregnancy (six to seven weeks of gestation). The relief of having a normal appearing girl is incalculable for those parents who have gone through the experience of having an infant with ambiguous genitalia who needs complex repeated surgical procedures. The benefit is not only immediate, but should also be evident in later life for reproductive function. Considerable emphasis has been placed on the low fertility rates reported in a population of patients with congenital adrenal hyperplasia centred on the Johns Hopkins Hospital in Baltimore. ${ }^{2}$ These were a heterogeneous group surveyed by questionnaire only and not monitored intensively by methods in common practice nowadays. A more recent study, but involving a smaller population based in Cardiff, reported an overall fertility rate of $60 \%$ in patients with the severe salt wasting form of congenital adrenal hyperplasia. ${ }^{3}$ It is apparent from these and other studies that heterosexual activities are influenced by the outcome of corrective surgery of the genitalia. The causes of reduced fertility in women with congenital adrenal hyperplasia are multiple and include low maternalism. ${ }^{4}$ Perhaps this latter observation is akin to the defeminised behaviour observed in childhood, ${ }^{5}$ phenomena attributed to prenatal androgen effects on the developing brain. Another feature that probably contributes to low fertility rates in congenital adrenal hyperplasia is the universal presence of polycystic changes in ovaries on ultrasound. ${ }^{6}$ Even though such changes are observed in about $20 \%$ of normal females, the propensity to develop polycystic ovarian syndrome and all the attendant morbidities must be greater for a woman with congenital adrenal hyperplasia. Thus, treating a female congenital adrenal hyperplasia fetus with antenatal steroids has a number of actual and potential benefits.

What of the potential dangers of antenatal treatment highlighted by Professor Brook in his review and debated in detail in a recent trio of papers in an endocrine journal. ${ }^{7-9}$ There is concern about the need to treat initially all pregnancies at risk until an affected female fetus is identified by prenatal testing. The danger of miscarriage as a result of the test procedures is emphasised, but such warnings are generic to fetal medicine practice. Amniotic fluid steroid analysis using specific chromatographic techniques on mid-trimester samples is the safest invasive test and very reliable. ${ }^{10}$ However, this approach does lengthen the time for treatment deemed unnecessary. The molecular genetics of 21-hydroxylase deficiency is complicated by phenomena such as transfer of genetic material from a pseudogene to an active gene by recombination events, gene duplication, uniparental disomy, and gonadal mosaicism. ${ }^{11}$ Nevertheless, a limited number of mutations account for most cases of 21-hydroxylase deficiency. A priori, the genotype of the index case and parents (obligate carriers) must be ascertained before contemplating antenatal treatment during a subsequent at risk pregnancy. The genotype predicts the phenotypic expression of 21-hydroxylase deficiency relatively well, unlike other single gene 
disorders which cause genital ambiguity such as the syndromes of androgen insensitivity. ${ }^{12}$ Certain mutations in the CYP21 gene are always associated with salt wasting and hence lead to severe masculinisation. ${ }^{13}$ Conversely, a few well defined mutations result in the mild late onset form of congenital adrenal hyperplasia, for which antenatal treatment would not be indicated. Although there will always be rare exceptions to the rule which usually offer fascinating insights into molecular mechanisms, ${ }^{14}{ }^{15}$ the review places undue emphasis on genetic curios.

Dexamethasone is currently the glucocorticoid of choice for antenatal treatment for several reasons. It circulates unbound and is not readily deactivated when it crosses the placenta by the $11 \beta$-hydroxysteroid dehydrogenase type 2 isoenzyme, which normally catalyses the cortisol-cortisone shuttle. ${ }^{16}$ Pharmacokinetic studies in patients with congenital adrenal hyperplasia indicate satisfactory oral bioavailability and a half life for dexamethasone of about three to four hours. ${ }^{17}$ It is advisable to administer the dose every eight hours. The quoted daily dose of $20 \mu \mathrm{g} / \mathrm{kg}$ was derived from the results of suppressed midtrimester amniotic fluid 17-hydroxyprogesterone concentrations. ${ }^{7}$ Maternal side effects can be excessive, and further studies are needed to determine the lowest dexamethasone dose that can adequately suppress excessive adrenal androgen secretion. The combination of changing maternal size and a lowering of the administered dose later in pregnancy should reduce the incidence of side effects.

What is the risk to the fetus from this treatment? In general, infants born to mothers who have received steroids during pregnancy for a variety of reasons are not subject to more congenital malformations or adverse effects on growth and development. There must be concern about the effect of dexamethasone on the early developing fetus, particularly when seven out of eight do not need the treatment. However, there is no evidence currently of measurable ill effects, either at birth or in later childhood. The experimental rat model of dexamethasone induced fetal growth retardation and later hypertension is of scientific interest, ${ }^{18}$ but the results cannot be directly related to the human situation, either for the newborn or for morbidity in adult life. The Swedish study of antenatal congenital adrenal hyperplasia treatment certainly contains the most informative outcome data so far reported. ${ }^{19}$ The paper describes adverse events in several treated infants, but most are the result of factors or specific disorders unrelated to steroid exposure. However, it is important not to be complacent just because treatment is successful in preventing abnormal genital development and in maintaining normal growth and development in most infants. Nevertheless, that medical treatment alone produces completely normal genitalia in a female infant with severe congenital adrenal hyperplasia is a major endocrine achievement. Indeed, $11 \beta$-hydroxylase deficiency, which is a form of congenital adrenal hyperplasia that causes the most profound masculinisation, has also now been successfully treated antenatally. ${ }^{20}$ The initial results of antenatal treatment for congenital adrenal hyperplasia are impressive. However, there must be a mechanism to ensure a coordinated approach on a national scale towards antenatal diagnosis, treatment, and subsequent monitoring long term for all infants exposed to dexamethasone in utero. Such an exercise will soon be underway through the auspices of the British Society for Paediatric Endocrinology and Diabetes, in collaboration with similar studies in other European countries.

Department of Paediatrics

IEUAN HUGHES

University of Cambridge

Addenbrooke's Hospital

Cambridge CB2 2QQ, UK

email:iah1000@cam.ac.uk

1 David M, Forest MG. Prenatal treatment of congenital adrenal hyperplasia resulting from 21-hydroxylase deficiency. F Pediatr 1984;105:799-801.

2 Mulaikal RM, Migeon CJ, Rock JA. Fertility rates in female patients with congenital adrenal hyperplasia due to 21-hydroxylase deficiency. $N$ Engl f Med 1987;316:17821-h.

3 Premawardhana LDKE, Hughes IA, Read GF, et al. Longer term outcome in females with congenital adrenal hyperplasia: the Cardiff experience. Clin Endocrinol (Oxf) 1997;46:327-32.

4 Meyer-Bahlburg HFL. What causes low rates of childbearing in congenital adrenal hyperplasia? $f$ Clin Endocrinol Metab 1999;84:1844-7.

5 Dittman RW, Kappes MH, Kappes ME, et al. Congenital adrenal hyperplasia. I. Gender-related behavior and attitudes in female patients and sisters. Psychoneuroendocrinology 1990;15:401-20.

6 Carmina E, Lobo RA. Polycystic ovary syndrome (PCOS): arguably the most common endocrinopathy is associated with significant morbidity in women. 7 Clin Endocrinol Metab 1999;84:1897-9.

7 Forest MG, Morel Y, David M. Prenatal treatment of congenital adrenal hyperplasia. TEM 1998;9:284-9.

8 Miller WL. Prenatal treatment of congenital adrenal hyperplasia: a promising experimental therapy of unproven safety. TEM 1998;9:290-3.

9 Ritzén EM. Prenatal treatment of congenital adrenal hyperplasia: a commentary. TEM 1998;9:293-5.

10 Wudy SA, Dorr HG, Solleder C, et al. Profiling steroid hormones in amniotic fluid of midpregnancy by routine stable isotope dilution/gas chromatographic-mass spectrometry: reference values and concentrations in fetuses at risk for reference values and concentrations in fetuses at risk for
21-hydroxylase deficiency. $\mathcal{F}$ Clin Endocrinol Metab 1999;84:2724-8.

11 López-Gutiérrez AU, Riba L, Ordoñez-Sánchez ML, et al. Uniparental disomy for chromosome 6 results in steroid 21-hydroxylase deficiency: evidence of different genetic mechanisms involved in the production of the disease. $f$ Med Genet 1998;35:1014-19.

12 Ahmed SF, Cheng A, Dovey L, et al. Phenotypic features, androgen receptor binding and mutational analysis is 278 clinical cases reported as androgen insensitivity syndrome. f Clin Endocrinol Metab 2000;(in press).

13 Jääskeläinen J, Levo A, Voutilainen R, et al. Population-wide evaluation of disease manifestation in relation to molecular
genotype in steroid 21-hydroxylase (CYP21) deficiency: good correlation in a well defined population. F Clin Endogood correlation in a well defin
crinol Metab 1997;82:3293-7.

14 Nimkarn S, Cerame BI, Wei J-Q, et al. Congenital adrenal hyperplasia without demonstrable genetic mutations. $\mathcal{f}$ Clin Endocrinol Metab 1999;84:378-81.

15 White PC. Comment on congenital adrenal hyperplasia without demonstrable genetic mutations [letter]. $\mathcal{F}$ Clin Endocrinol Metab 1999;84:2976.

16 Edwards CRW, Benediktsson R, Lindsay R, et al. Dysfunction of the placental glucocorticoid barrier: a link between the foetal environment and adult hypertension? Lancet 1993;341:938-41.

17 Young MC, Cook N, Read GF, et al. The pharmacokinetics of low-dose dexamethasone in congenital adrenal hyperplasia. Eur f Clin Pharmacol 1989;37:75-7.

18 Benediktsson R, Lindsay R, Noble J, et al. Glucocorticoid exposure in utero: a new model for adult hypertension. Lancet 1993;341:339-41.

19 Lajic S, Wedell A, Bui T-H, et al. Long-term somatic follow-up of prenatally treated children with congenital adrenal hyperplasia. $f$ Clin Endocrinol Metab 1998;83:3872-80.

20 Cerame BI, Newfield RS, Pascoe L, et al. Prenatal diagnosis and treatment of $11 \beta$-hydroxylase deficiency congenital adrenal hyperplasia resulting in normal female genitalia. $\mathcal{f}$ Clin Endocrinol Metab 1999;84:3129-34. 


\section{Commentary}

There can be few situations more worrying to new parents than ambiguous genitalia in their newborn baby, with the uncertainty of sex and diagnosis until tests have been carried out. The commonest cause is 21-hydroxylase deficiency congenital adrenal hyperplasia in an affected female infant. In this situation, there is the prospect of surgery (perhaps repeatedly) in infancy, and sometimes at adolescence, and concerns about fertility and psychosexual orientation and functioning.

Thus when there has been a previously affected female child, the possibility of prenatal treatment to reduce or prevent significant neonatal virilisation in a second affected female fetus and obviate the need for surgery in infancy and beyond is potentially attractive.

Prenatal treatment with the potent and long acting synthetic glucocorticoid dexamethasone has been available and administered for over 15 years, ${ }^{12}$ and an algorithm for the prenatal management of the "at risk" pregnancy has been published. ${ }^{3}$ What is an appropriate dose? What is the evidence that it is effective in terms of reducing virilisation significantly? Could there be positive effects on psychosexual orientation or fertility? Is it safe for the mother? What and how significant are potential side effects in the fetus and on the subsequent development of the child or even adult?

Fetuses in whom treatment would be appropriate are affected females, a one in eight possibility with each pregnancy. However, dexamethasone must be started very early in pregnancy, before sexual differentiation starts at six weeks gestation and considerably before antenatal diagnosis is possible. Thus seven out of eight infants will have treatment unnecessarily for a number of weeks. In affected female infants, treatment is continued to term.

Important outcomes have not been comprehensively evaluated in significant numbers of pregnancies, but several large scale studies have now been reported in varying detail..$^{4-8}$ Maternal complications have included increased appetite, weight gain (sometimes extreme), oedema, hypertension, glucose intolerance, mood swings, epigastric pain, facial hirsutism and Cushingoid features (including moon-face, plethora with permanent scarring), and spontaneous miscarriage. The reasons for the differing reported incidence of such problems are as yet unresolved. There is possibly an unexplained/unexpected fetal miscarriage rate of up to $1 \%$ at or near term.

All 44 prenatally treated children in Sweden and Norway have been studied at birth and followed up for between one and 10 years. $^{78}$ Short term dexamethasone treatment produced no difference in growth (weight and length/height) at birth or subsequently compared with controls. Although six affected girls treated throughout gestation showed appreciably reduced virilisation compared with their elder sisters, in the cohort of treated children there was a substantial number of unexpected fetal problems including hydrocephalus, agenesis of the corpus callosum, mental retardation and ataxia, and a mitochondrial disorder. In the matched control group, there was one child with Down syndrome.

A causal relation between dexamethasone treatment and the adverse outcomes is uncertain but gives considerable concern. Doses of dexamethasone used are very high - as they are, for example, in the treatment of preterm infants with chronic lung disease. In the latter situation, there are reports of short term reductions in weight gain, linear growth, and lower leg growth, ${ }^{910}$ although the longer term effects on growth are more controversial. ${ }^{11}{ }^{12}$ Importantly, neurodevelopmental impairment may be increased if treatment is started within the first four postnatal days. ${ }^{13}$

Our track record in perinatology with the introduction of unproven treatments is not good. Silverman ${ }^{14}$ has reported that of 25 treatments in neonatology introduced over his professional lifetime, four have led to improved practice, 12 have misled into fruitless byways, and nine have led to disaster.

$11 \beta$-Hydroxysteroid dehydrogenase is plentiful in the placenta and could be important in protecting the fetus from maternal glucocorticoid. In rats, increased $11 \beta$-hydroxysteroid dehydrogenase activity is associated with increased fetal weight with large placentae. The fetal rat treated with dexamethasone is growth retarded and hypertensive as an adult. $^{15}$ Epidemiological evidence suggests that small fetuses are more likely to develop hypertension, other cardiovascular diseases, and type 2 diabetes mellitus in adulthood. ${ }^{16}$ It is speculated that the growth retarded human fetus has been exposed to excessive glucocorticoid in utero because of relative placental $11 \beta$-hydroxysteroid dehydrogenase deficiency, which could reflect placental and fetal growth, and that this has long lasting effects-for example, adult hypertension-by imprinting through a brain receptor or neurochemical mechanisms. ${ }^{17}$

Dexamethasone seems generally to be effective at reducing virilisation, but only to the extent of preventing the need for surgery in about $50 \% .^{5}$ Variation in the degree of virilisation in successive affected infants, whether treated with glucocorticoid or not, can be considerable. Potential long term deleterious effects on, for example, childhood brain growth and development or bone mineralisation have not been adequately studied. However, nor have (potentially beneficial) effects on psychosexual orientation, given the likely role of fetal androgens in imprinting male gender identity. ${ }^{18} 19$

A formal systematic review of the relevant literature is currently in progress. However, it is already clear that the data reported so far, worrying as they are, are for relatively small numbers from observational studies, with no randomisation or placebo control groups, and are likely to be considerably biased. Nor do they look systematically at all relevant areas of concern, which must now include blood pressure, cardiovascular risk factors, somatic and brain growth, psychosexual orientation, 
and sexual and psychological functioning and fertility.

In this, as in a other areas of paediatric endocrinology, there is an urgent need for large well constructed clinical trials to ensure accurate results with statistical and clinical significance. There must be more collaboration between centres, both nationally and internationally, to design prospective controlled multicentre trials which can recruit sufficient numbers for meaningful efficacy and safety outcomes to be obtained over a long period of time; all perinatal trials should report long term morbidity. ${ }^{20}$

There still remains insufficient evidence on the appropriate dosage regimen, safety in the mother, and short and long term safety and efficacy in the fetus from appropriately controlled studies to recommend the use of dexamethasone outwith the context of such controlled scientific studies.

CHRISTOPHER J H KELNAR

Senior Lecturer

Department of Child Life and Health, University of Edinburgh

email:dkcantavark@dial.pipex.com

1 David M, Forest MG. Prenatal treatment of congenital adrenal hyperplasia resulting from 21-hydroxylase deficiency. F Paediatr 1984;105:799-801.

2 Evans MI, Chrousos GP, Mann DW, et al. Pharmacologic suppression of the fetal adrenal in utero. $f A M A$ 1985;253:1015-20.

3 New MI, Ghizzoni L, Speiser PW. Update on congenital hyperplasia. In: Lifshitz F, ed. Pediatric endocrinology. New York: Marcel Dekker, 1996:305-20.

4 Forest MG, Dörr HG. Prenatal treatment of congenital adrenal hyperplasia due to 21-hydroxylase activity: European experience in 223 pregnancies at risk. Pediatr Res 1993;33(suppl 3):abstract 10 .
5 Mercado AB, Wilson RC, Cheng KC, Wei J-Q, New MI Prenatal treatment and diagnosis of congenital adrenal
hyperplasia owing to steroid 21 -hydroxylase deficiency. $\mathcal{F}$ Clin Endocrinol Metab 1995;80:2014-20.

6 Levine LS, Pang S. Prenatal diagnosis and treatment of congenital adrenal hyperplasia. I Pediatr Endocrinol 1994;7:193-200.

7 Lajic S, Wedell A, Bui T-H, Ritzén EM, Holst M. Long term somatic follow-up of previously treated children with CAH. 7 Clin Endocrinol Metab 1998;83:3872-80.

8 Ritzén M, Lajic S, Wedell A. Congenital adrenal hyperplasia due to 21-hydroxylase deficiency [supplement]. Horm Res 1999:(in press).

9 Gibson AT, Pearse RG, Wales JKH. Growth retardation after dexamethasone administration: assessment by knemometry. Arch Dis Child 1993;69:505-9.

10 Weiler HA, Paes B, Shah JK, Atkinson SA. Longitudinal assessment of growth and bone mineral accretion in prematurely born infants treated for chronic lung disease. Early Hum Dev 1997;47:271-86.

11 Jones R, Wincott E, Elbourne D, Grant A. Controlled trial of dexamethasone in neonatal chronic lung disease: a 3 year follow-up. Pediatrics 1995;96:897-906.

12 Yeh TF, Lin YJ, Huang CC, et al. Early dexamethasone therapy in preterm infants: a follow-up study. Pediatrics 1998;101:E7.

13 Halliday HL. Clinical trials of postnatal corticosteroids: inhaled and systemic. Biol Neonate 1999;76:(suppl 1): $29-40$.

14 Silverman WA. Retrolental fibroplasia: a modern parable. Monographs in neonatology. New York:Grune and Stratton, 1980.

15 Benediktsson R, Lindsay R, Noble J, Seckl JR, Edwards CRW. Glucocorticoid exposure in utero: a new model for CRW. Glucocorticoid exposure in utero: a ne
adult hypertension. Lancet 1993;341:339-41.

16 Barker DJP. Fetal and infant origins of adult disease. London: BMJ Publishing Group, 1992.

17 Edwards CRW, Benediktsson R, Lindsay R, Seckl JR. Dysfunction of the placental gluccorticoid barrier: link between fetal environment and adult hypertension. Lancet 1993;341:355-7.

18 Imperato-McGinley J, Peterson RE, Gautier T, Sturla E. Androgens and the evolution of gender identity among male pseudohermaphrodites with 5-alpha-reductase defimale pseudohermaphrodites with 5-alp.

19 Imperato-McGinley J, Peterson RE, Stoller R, Goodwin WE. Male pseudohermaphroditism secondary to 17-betahydroxysteroid dehydrogenase deficiency: gender role change with puberty. $\mathcal{f}$ Clin Endocrinol Metab 1979;49:291-5.

20 Johnson A. Randomised controlled trials in perinatal medicine. 3. Identifying and measuring endpoints in randomised controlled trials. $\mathrm{Br} \mathcal{F}$ Obstet Gynaecol 1997;104:768-71. 